\title{
Educação Permanente em Saúde: uma estratégia para refletir sobre o processo de trabalho
}

Janaína Rocha de Sousa Almeida*; Davi Oliveira Bizerril*; Kátia de Góis Holanda Saldanha**; Maria Eneide Leitão de Almeida***

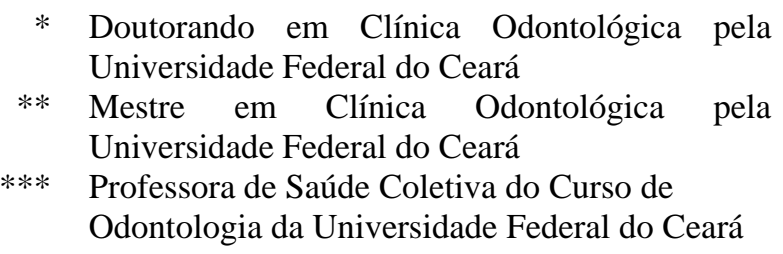

\section{RESUMO}

A Educação Permanente em Saúde (EPS) é aprendizagem no trabalho, onde aprender e ensinar incorporam-se às organizações e processos de trabalho. Assim, iniciou-se uma proposta de EPS no município de Fortaleza/CE, objetivando induzir mudanças nas diversas maneiras de agir dos profissionais da Estratégia de Saúde da Família (ESF). Foi realizado um curso de atualização em Saúde Bucal para trezentos cirurgiões dentistas (CD). Trata-se de um relato de experiência, onde são descritos planejamento, execução e avaliação do curso, que foi dividido em dois módulos: Atenção Primária e Secundária em Saúde. Concluíram o curso 207 CD, 69\% dos matriculados. Como resultados, obteve-se a construção do Manual de Promoção em Saúde Bucal e do Manual de Encaminhamentos para a Atenção Secundária em Odontologia. As ações de EPS são importantes para os profissionais de saúde, motivando-os diante dos desafios do cotidiano.

Descritores: Estratégia Saúde da Família. Saúde Bucal. Educação Continuada.

\section{INTRODUÇÃO}

A criação do Sistema Único de Saúde (SUS) tornou evidente a necessidade de mudança na formação dos profissionais de saúde. No Brasil esses profissionais são formados com grande domínio de técnicas, porém diversas vezes são incapazes de compreender a subjetividade e a diversidade da cultura das pessoas. Não são preparados para o trabalho de forma multi e interdisciplinar e apresentam dificuldades de implementar os princípios do SUS no seu serviço diário ${ }^{1}$.

Espera-se que a formação dos novos profissionais de saúde seja voltada à realidade da população, respeitando suas necessidades com um modelo de assistência de excelência $^{2,3}$. Ressalta-se que a reorientação da formação profissional em saúde deve ser um movimento de fortalecimento do SUS, portanto, a formação em serviço passa a ter papel fundamental na metodologia pedagógica.

De acordo com a Constituição Brasileira, em seu artigo 200, é dever do estado ordenar a formação de recursos humanos na área da saúde ${ }^{4}$. Com isso, em 13 de fevereiro de 2004 através da portaria 198GM foi implementada a Política Nacional de Educação Permanente em Saúde como estratégia do Sistema Único de Saúde para a formação e o desenvolvimento de trabalhadores para o setor ${ }^{2,5}$.

A educação permanente é uma proposta 
político-pedagógica que favorece, aos trabalhadores, um processo de ensinoaprendizagem dentro do seu cotidiano laboral. Tal processo defende uma filosofia de reflexão e crítica sobre os processos de trabalho dos profissionais ${ }^{6}$. Os processos de qualificação dos trabalhadores de saúde devem ter como referência as necessidades da população, da gestão e do controle social ${ }^{7}$. É importante que tenham como objetivos a transformação das práticas profissionais e da própria organização do trabalho sendo estruturados a partir da problematização do processo de trabalho ${ }^{8,9}$.

Esta Educação Permanente em Saúde destaca-se pela valorização do trabalho como fonte do conhecimento, vinculação do cotidiano e do processo de aprendizagem, orientação das ações educativas para a integração do trabalho de maneira inter e multiprofissional ${ }^{10}$. Com isso, a educação deve ser trabalhada de maneira permanente e dinâmica, buscando construir espaços coletivos para a reflexão e avaliação, pondo o cotidiano do trabalho em análise ${ }^{11}$.

Através da discussão sobre o cotidiano de trabalho, a educação permanente em saúde possibilita pactos e acordos coletivos de trabalho no SUS, seu foco são os processos de trabalho, seu alvo são as equipes e seu lócus de produção são os coletivos $^{8}$. Essa aprendizagem dos profissionais de saúde passa a ter sentido quando ela é significativa, ou seja, quando o material a ser aprendido tem algum sentido para o educando ${ }^{1}$.

Diante da necessidade de desenvolver um processo de formação permanente dos profissionais de saúde bucal, a proposta desse artigo foi relatar a experiência do Curso de Atualização em Saúde Bucal na Estratégia de Saúde da Família (ESF), desde sua estruturação até a finalização com seus produtos e reflexões.

\section{RELATO DE EXPERIÊNCIA}

Neste relato de experiência serão abordados os aspectos relacionados ao planejamento, execução e avaliação de atividade de educação permanente em saúde bucal na Estratégia de Saúde da Família.

O público alvo da ação foram todos os cirurgiões-dentistas do município de Fortaleza/CE, totalizando 300 profissionais. A atividade de educação permanente teve uma carga horária de 80 horas, com periodicidade semanal e duração de 6 meses, no período de outubro de 2013 a março de 2014. Foram ofertadas 6 turmas, cada uma com cerca de 50 alunos.

Os facilitadores das atividades foram os próprios profissionais $\mathrm{CD}$ da Estratégia de Saúde da Família e dos Centros de Especialidades Odontológicas, totalizando uma quantidade de 24 facilitadores, o que permitiu uma discussão totalmente baseada na realidade dos serviços.

A metodologia utilizada para o desenvolvimento da atividade foi a problematização, que tem seus fundamentos filosóficos baseados no referencial teórico de Paulo Freire. A educação problematizadora fundamenta-se na relação dialógica entre educando e educador, onde ambos aprendem juntos, visto que a educação não é uma prática de depósitos de conteúdos apoiada numa concepção de seres vazios. A problematização trabalha a construção de conhecimentos a partir da vivência de experiências significativas, sendo o conteúdo oferecido na forma de problemas e não de forma acabada, problemas esses que devem ser discutidos pelos alunos ${ }^{12}$. Visando a implantação dessa metodologia, propôs-se a construção do conhecimento através do movimento de agir sobre a realidade, orientando-se desta maneira o sujeito pela observação da práxis. Neste modelo o diálogo é essencial, os problemas estudados necessitam de um espaço real, 
assim a construção do conhecimento acontece de forma significativa ${ }^{13,14}$.

O curso foi dividido em dois módulos: o primeiro abordou a temática da Atenção Primária em Saúde e o segundo Atenção Secundária em Saúde. No primeiro módulo foi proposto a inclusão temas: a Política Nacional de Humanização com foco no Acolhimento e Estratificação de Risco em Saúde Bucal, Ações de Promoção de Saúde Bucal, Programa de Saúde na Escola (PSE), Programa Nacional de Melhoria do Acesso e Qualidade (PMAQ), Contrato Organizativo de Ação Pública (COAP) e Farmacologia (figura 1).

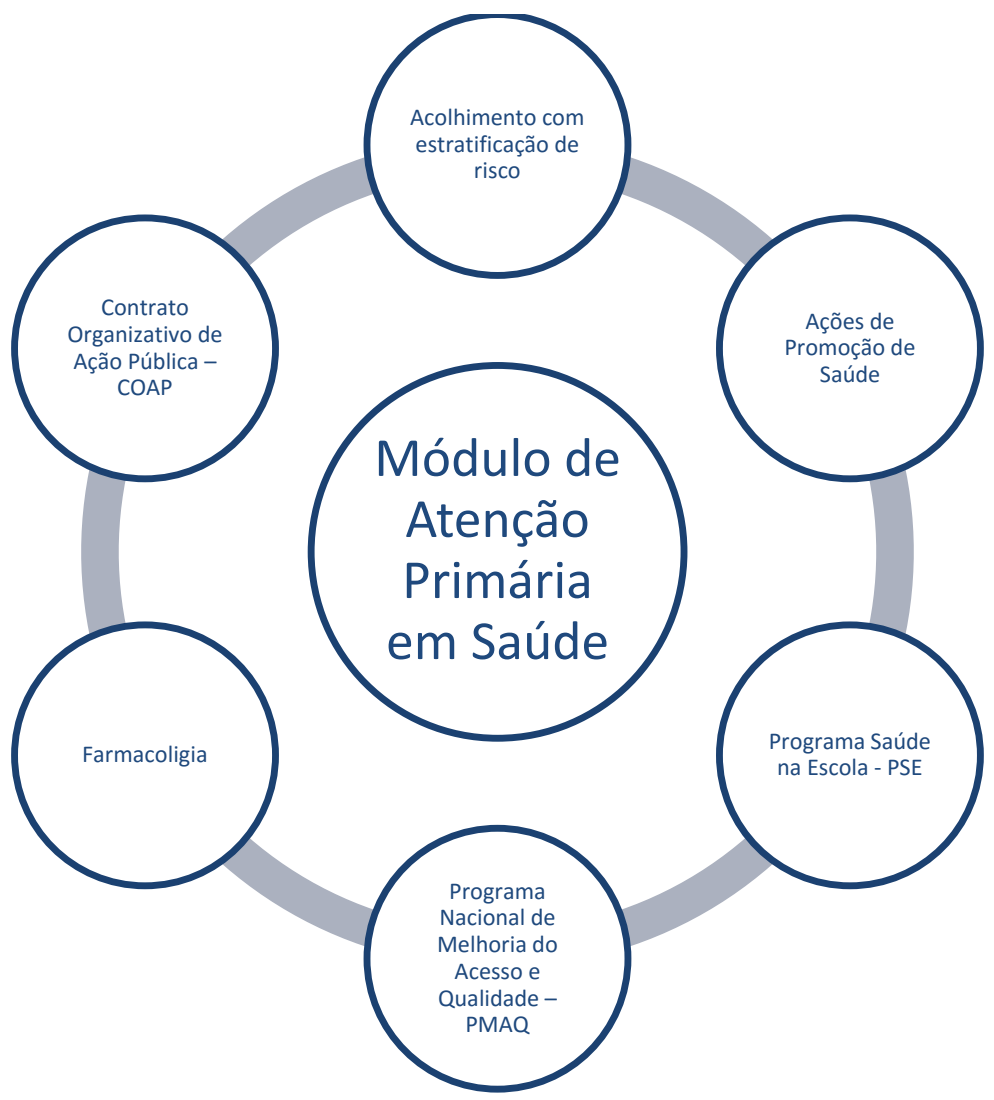

Figura 1. Módulo de Atenção Primária em Saúde. Atividade de Educação Permanente em Saúde para os cirurgiões dentistas do município de Fortaleza/CE.

A oficina sobre a Política Nacional de Humanização do Sistema Único de Saúde enfatizou o acolhimento com estratificação de risco em saúde bucal, utilizado pelas equipes de saúde bucal do município de Fortaleza. Discutiu-se a questão do acesso dos usuários com necessidade de tratamento odontológico, buscando-se a melhor maneira de oferecer esse serviço a maior parte dos usuários, utilizando-se como premissa o princípio da equidade, onde os que tem mais necessidade são agendados primeiro.
Historicamente o trabalho do CD está atrelado ao equipamento odontológico, porém sabe-se que suas funções vão além disso. A discussão sobre as ações de promoção de saúde bucal aconteceu nesta perspectiva, enfatizando-se a promoção e prevenção da saúde dos usuários do SUS. Seguindo-se as discussões sobre promoção de saúde, o Programa de Saúde na Escola (PSE) foi trabalhado, sendo esta uma política intersetorial da saúde e da educação voltada às crianças, adolescentes, jovens e adultos da 
educação pública brasileira, que integra os profissionais da Estratégia de Saúde da Família e profissionais da educação ${ }^{15}$.

Buscando-se a discussão sobre o acesso e a qualidade do serviço debateu-se sobre o Programa Nacional de Melhoria do Acesso e Qualidade (PMAQ), programa de âmbito nacional que tem como objetivo promover a melhoria do acesso e da qualidade da atenção à saúde. Lançado em 2011, o PMAQ da Atenção Básica (PMAQ-AB) contemplou em seu primeiro ciclo a adesão de equipes de Saúde da Família e de Atenção Básica parametrizadas, incluindo equipes de saúde bucal $^{15}$. A discussão sobre o PMAQ objetivou esclarecer as dúvidas dos profissionais $\mathrm{e}$ problematizar como este poderia ser utilizado para melhorar e potencializar os serviços de saúde.

Compreendendo que é importante o conhecimento das metas pactuadas dentro dos serviços de saúde problematizou-se a temática do Contrato Organizativo de Ação Pública COAP, sendo este um acordo de colaboração firmado entre os três entes federativos, no âmbito de uma região de saúde, com o objetivo de integrar as ações e serviços de saúde e garantir a integralidade da atenção à saúde da população no território ${ }^{16}$.

Dentre as dificuldades clínicas na Atenção Primária em Saúde, destacam-se os enfrentamentos vivenciados no campo da farmacologia, quer pela falta de medicamentos disponíveis ou o uso inadequado por parte dos usuários, dentre outros, produzindo reflexões sobre o uso racional de medicamentos disponíveis nas farmácias das Unidades de Saúde.

No módulo de Atenção Secundária em Saúde, como estratégia de intervenção, realizou-se um planejamento das oficinas com os especialistas que atendem nos Centros de Especialidades Odontológicas do município.
De acordo com as demandas apresentadas pelos $\mathrm{CD}$ do referido município, as quais estavam relacionadas aos atendimentos clínicos diários e referentes aos encaminhamentos para a Atenção Secundária, elaborou-se casos clínicos nas especialidades de Estomatologia, Cirurgia Bucomaxilofacial, Odontopediatria, Periodontia, Endodontia, Prótese Dentária, Disfunção Temporomandibular e Pacientes com Necessidades Especiais. A partir daí, desenvolveram-se as discussões baseadas na experiência do próprio serviço (figura 2).

Ao final do módulo de Atenção Secundária, ocorreu um momento de dispersão no qual foi proposto aos participantes que formulassem uma atividade de promoção de saúde destinada aos usuários do seu território de atuação e refletissem sobre sua própria prática, trazendo o relato dessa experiência para compartilhar com todo o grupo de alunos. Essa atividade de promoção de saúde poderia ser desenvolvida dentro da Unidade de Atenção Primária em Saúde ou no território.

Esse curso de atualização estava proposto como parte da contribuição de pesquisa aprovada pelo comitê de ética em pesquisa em 10/02/2014, com o parecer $n^{\circ}$ 526.915 e que foi conduzido conforme os princípios éticos da Declaração de Helsinque, contidos na resolução $\mathrm{n}^{\circ} 466$ em 12 de dezembro de 2012, do Conselho Nacional de Saúde, que regula investigações realizadas em seres humanos.

Concluíram o curso 207 cirurgiõesdentistas, $69 \%$ dos matriculados. O curso foi disponibilizado para todos os cirurgiões dentistas do município o que proporcionou uma interação entre profissionais da atenção primária e secundária, possibilitando uma discussão ampla sobre os processos de trabalho. 


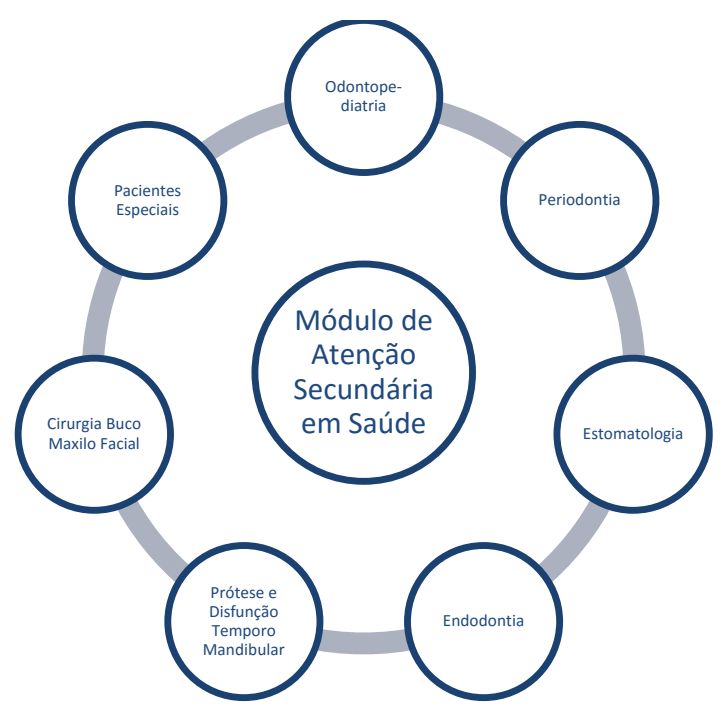

Figura 2. Módulo de Atenção Secundária em Saúde. Atividade de Educação Permanente em Saúde para os cirurgiões dentistas do município de Fortaleza/CE.

$\mathrm{O}$ acolhimento com estratificação de risco em saúde bucal passou a ser utilizado no município como forma de organizar o acesso dos usuários ao serviço de odontologia, utilizando-se o princípio da equidade como regulador do agendamento odontológico.

A participação de representantes da secretaria de educação na temática do PSE resultou na melhor articulação das ações desenvolvidas nas escolas pelas equipes de saúde da família.

Como produtos do referido curso obteve-se um Manual de Promoção de Saúde Bucal com relatos de ações desenvolvidas pelos cirurgiões dentistas do município que servirá como um guia para a reprodução das ações de promoção de saúde em seus territórios. Ações essas que podem ser realizadas com os mais diversos grupos etários como crianças, adolescentes, gestantes, adultos e idosos. Podendo também desenvolver atividades de levantamentos epidemiológicos com finalidades de organização do processo de trabalho e busca de lesões potencialmente cancerizáveis.

As discussões sobre os procedimentos da atenção secundária resultaram na elaboração de um Manual de Encaminhamentos para a Atenção Secundária em Odontologia - Centros de Especialidades Odontológicas. Este manual visa evitar os encaminhamentos errôneos para a atenção secundária e contém as orientações necessárias para que o usuário seja encaminhado de maneira correta, tornando seu tratamento mais rápido e eficiente.

\section{DISCUSSÃO}

A taxa de evasão encontrada de $31 \%$ mostra-se semelhante ao observado por Carvalho $(2011)^{7}$, onde dois terços dos profissionais concluíram o curso de educação permanente em saúde.

A educação permanente em saúde é fundamental para a efetivação de um serviço de saúde de qualidade e excelência. Para que isto aconteça é necessário a compreensão de todo o processo de trabalho buscando competências para atuar com os problemas de saúde em contextos socioculturais variado $^{17,18}$. Nesse sentindo, a Política Nacional de Educação Permanente em Saúde 
significa um espaço de discussões onde todos os atores envolvidos possuem sua importância e contribuem para a ressignificação dos processos de saúde. A participação dos próprios cirurgiões dentistas do município de Fortaleza/CE como facilitadores do processo de educação permanente possibilitou uma valorização dos profissionais, bem como contribuiu na construção de uma aprendizagem significativa. $\mathrm{Na}$ proposta da educação permanente o professor passa a ter o papel de facilitador da aprendizagem, possibilitando a construção de conhecimentos com significado para a prática diária dos profissionais de saúde. ${ }^{19}$

Sendo assim, o processo de ensino e aprendizagem não deve restringir-se a transferência de normas e protocolos, deve-se destacar a experiência profissional e a experiência pessoal deve ser considerada. A bagagem de conhecimentos que o indivíduo traz deve ser considerada e agregada ao desenvolvimento de um serviço de qualidade $^{19,20}$. Portanto, a participação dos profissionais durante este curso foi construtiva, onde foi possível não apenas apresentar protocolos pré-estabelecidos, mas construí-los de acordo com as necessidades dos profissionais, gestão e usuários.

A educação permanente sendo a aprendizagem no trabalho, incorpora o aprender e o ensinar ao cotidiano, estando fundamentada em diferentes vertentes metodológicas. Destaca-se aqui as contribuições de Paulo Freire, onde a problematização considera os conhecimentos e experiências que as pessoas já possuem $^{9,20,22,23}$, assim como foi realizado nesta atividade, onde todas as discussões foram baseadas em casos reais presentes no cotidiano dos profissionais, o que tornou a ação mais interessante e construtiva.

No processo de acolhimento destacou- se a estratificação de risco dos usuários, onde foram estabelecidos graus de prioridade para $\mathrm{o}$ atendimento odontológico, de acordo com o princípio da equidade, sendo padronizada essa orientação para o agendamento odontológico. A utilização do princípio da equidade para a organização do atendimento odontológico também pode ser verificada no estado de Minas Gerais ${ }^{24}$ e Paraná ${ }^{25}$. A proposta de estratificação de risco apresentada foi alvo de crítica de alguns profissionais que compreendem a necessidade de universalizar o atendimento, porém a cobertura de saúde bucal do município de apenas 36\%, impossibilita a universalização, tornando-se necessário buscar o princípio da equidade para organizar o serviço de saúde bucal.

Associando-se as questões clínicas com as atividades de promoção de saúde, destacase o PSE ${ }^{26,27}$. Foi importante a discussão sobre o papel dos profissionais da ESF frente ao desenvolvimento deste programa e a importância da articulação da secretaria de saúde com a secretaria de educação. Destacase que os cirurgiões dentistas do município de Fortaleza apresentam um histórico positivo de articulação com os estabelecimentos de educação, desenvolvendo atividades de promoção de saúde nas escolas e creches, porém com o advento do PSE toda a equipe da ESF pode participar do desenvolvimento dessas ações.

Com relação ao Programa Nacional de Melhoria do Acesso e da Qualidade da Atenção Básica (PMAQ-AB) destaca-se que seu objetivo é instigar a ampliação do acesso e a melhoria da qualidade da atenção primária de saúde, com garantia de um padrão de qualidade comparável nacional, regional e localmente, de maneira a permitir maior transparência e efetividade das ações governamentais direcionadas à atenção primária à saúde ${ }^{28}$. Portanto, é de suma 
importância que todos os cirurgiões dentistas estejam cientes da finalidade deste programa e compreendam que o PMAQ não é um programa que de forma pontual, mas um processo contínuo de avaliação visando aperfeiçoar os processos, melhorar a qualidade e aumentar o acesso à população. A discussão sobre todos os indicadores do PMAQ, mostrou qual o objetivo de cada um e como devem ser calculados, a compreensão sobre o que é produzido torna a informação dos dados significativa sendo possível uma melhor avaliação sobre a qualidade do serviço prestado à população.

Com relação as discussões sobre o COAP, percebeu-se que muitos profissionais da ESF desconheciam seu significado e importância, o que foi trabalhado através da problematização consolidando conceitos e reflexões sobre o tema. Os profissionais compreenderam que o COAP tem o objetivo de garantir uma gestão compartilhada com segurança jurídica, transparência e solidariedade entre os entes federativos, buscando a garantia da efetividade do direito à saúde da população brasileira ${ }^{16}$.

A aprendizagem ativa descrita nesse artigo caracteriza-se pela construção de narrativas pelos alunos após o contato com a realidade, o que foi verificado através da construção do manual de promoção de saúde bucal, onde tem-se o relato de atividades de promoção e prevenção de saúde. A narrativa proporciona o encontro consigo mesmo e com o outro, podendo revelar um modo de viver na realidade. Houve diversidade de práticas consideradas exitosas e potentes para promoção da saúde, portanto, a construção de um manual com as referidas ações objetiva corroborar com a reprodução das mesmas por todos os profissionais da estratégia de saúde da família. As atividades utilizam metodologias ativas o que as torna mais interessante e atraentes para os participantes.

Contudo, é importante ressaltar que no SUS os serviços devem proporcionar a integralidade dos tratamentos de seus usuários, ancorando-se no entendimento e aplicação dos conceitos de referência e contrarreferência. A organização de um adequado sistema de referência à atenção especializada ainda representa um dos mais importantes desafios a serem enfrentados e evidencia, no cotidiano dos serviços, a busca por uma assistência integral aos $\operatorname{cidadãos}^{28}$. Considerando a realidade local, foram discutidos os princípios que deveriam ser seguidos para realizar as referências da melhor forma possível. A referência é representada pelo fluxo, por meio de encaminhamento do paciente com necessidades de saúde complexas para centros de atenção especializada (CEO). O retorno do paciente ao nível da atenção primária próxima de sua residência é denominado contrarreferência. $\mathrm{O}$ estabelecimento de pactuações para facilitar esse fluxo é primordial para que se faça um atendimento integral ${ }^{29}$. Essa pactuação foi realizada com os cirurgiões dentistas através desta atividade de educação permanente em saúde. A ação foi importante ao discutir casos que poderiam ser atendidos na atenção primária, sem a necessidade de encaminhar desnecessáriamente pacientes para os CEO, organizando da melhor forma o serviço odontológico.

\section{CONSIDERAÇÕES FINAIS}

Os objetivos de aprendizagem do curso foram atingidos visto que houve uma ampla discussão sobre os diversos temas abordados sempre os relacionando com os processos de trabalho, o que torna o resultado da atividade satisfatório.

A metodologia problematizadora foi o grande diferencial deste curso e trouxe mais interesse na participação por parte dos alunos. 
Os facilitadores eram os profissionais da rede de saúde e isso tornou de fato a aprendizagem significativa.

Dentre as dificuldades encontradas destacase o grande número de alunos o que necessitou uma logística pedagógica bem estruturada e organizada. Sugere-se que os próximos cursos tenham um menor número de participantes o que torna a metodologia problematizadora mais efetiva

As ações de educação permanente devem ser constantes nos serviços de saúde, contribuindo desta maneira para a formação de profissionais mais qualificados para o exercício de suas funções no SUS.

\begin{abstract}
Permanent Health Education: a strategy for reflection on the process of work

The Permanent Health Education (EPS) is learning at work, where learning and teaching are incorporated into the organization and work processes. Thus began an EPS proposal in Fortaleza/CE, aiming to induce changes in the various courses of action of the professionals of the Family Health Strategy (FHS). A refresher course in Oral Health for three hundred Dental General Practioners (DGP) was performed. This is an experience report that describes planning, implementation and evaluation of the course, which was divided into two modules: Primary and Secondary Health, concluded the course $207 \mathrm{CD}$, $69 \%$ of enrolled. As a result, we obtained the construction of the Promotion Manual on Oral Health and Referral Guide for Secondary Care in Dentistry. EPS actions are important for health professionals, motivating them before the everyday challenges.
\end{abstract}

Descriptors: Family Health Strategy. Oral Health. Continuing Education.

\section{REFERÊNCIAS}

1. Cardoso IM. "Rodas de Educação permanente" na Atenção Básica de saúde: analisando contribuições. Saúde São Paulo. 2012; 21:18-28.

2. Mendonça FF, Nunes EFPA. Necessidades e dificuldades de tutores e facilitadores para implementar a política de educação permanente em saúde em um município de grande porte no estado do Paraná, Brasil.
Interface (Botucatu). 2011; 15(38):871-82.

3. Noro LRA, Santos BCSF, Souza PHS, Pinheiro IAG, Borges REA, Nunes LMF, Cruz RCS, Silva SM. O professor (ainda) no centro do processo de ensino-aprendizagem em odontologia. Rev ABENO. 2015; 15(1):211.

4. Constituição (1988). Constituição da República Federativa do Brasil (1988). Presidência da República. Casa Civil. Subchefia para assuntos jurídicos. BrasíliaDF: Senado Federal; 1988.

5. Portaria $\mathrm{N}^{\circ} 198$, de 13 de fevereiro de 2004. Dispõe sobre a Política Nacional de Educação Permanente em Saúde. Diário Oficial da União, 13 de fev de 2004.

6. Garcia RM; Baptista R. Educação a distância para a qualificação dos profissionais do SUS: perspectivas e desafios. Rev Baiana de Saúde Pública. 2007; 31(Supl.1):70-78.

7. Carvalho BG, Turini B, Nunes EFPA, Bandeira IF, Barbosa PFA, Takao TS. Percepção dos médicos sobre o curso Facilitadores de Educação Permanente em Saúde. Rev Brasil de Educação Médica. 2011; 35(1): 132-41.

8. Brasil. Política Nacional de Educação Permanente em Saúde. Brasil. Ministério da Saúde. Secretaria de Gestão do Trabalho e da Educação na Saúde. Departamento de Gestão da Educação em Saúde. Brasília: Ministério da Saúde; 2009.

9. Falkenberg MB, Mendes TPL, Moraes EP, Souza EM. Educação em saúde e educação na saúde: conceitos e implicações para a saúde coletiva. Cienc Saude Colet. 2014; 19(3):84752.

10. Montanha M, Peduzzi M. Educação Permanente em Enfermagem: levanta-mento de necessidades e resultados esperados segundo a concepção dos trabalhadores. Rev. Esc Enferm USP. 2010; 44(3):597-604.

11. Ceccim RB, Capazzolo AA. Educação dos profissionais de saúde e afirmação da vida: a prática clínica como resistência. In: Marins JJN et al Educação Médica em Transformação. São Paulo: Abem - 
HUCITEC; 2004.

12. Cyrino EG, Pereira MLT. Trabalhando com estratégias de ensino aprendizado por descoberta na área da saúde: a problematização e a aprendizagem baseada em problemas. Cad Saúde Pública. 2004; 20(3): 780-88.

13. Sobral FR, Campos CJG. Utilização de metodologia ativa no ensino e assistência de enfermagem na produção nacional: revisão integrativa. Rev Esc Enferm USP. 2012; 46(1):208-18.

14. Marin MJS. Pós-graduação multi-profissional em saúde: relato de experiências utilizando metodologias ativas. Interface (Botucatu). 2010; 14(33):331-41.

15. Brasil. Política Saúde na Escola. Ministério da Saúde, Brasil. Departamento da Atenção Básica. Brasília: Ministério da Saúde; 2014. Acessado em 14 de dezembro de 2014. Disponível em: http://dab.saude.gov.br/ portaldab/pse.php

16. Brasil. Contrato Organizativo da Ação Pública da Saúde. Secretaria da Gestão Estratégica e Participativa. Brasília: Ministério da Saúde; 2011. Acessado em 31 de dezembro de 2015. Disponível em: http://bvsms.saude.gov.br

17. Peduzzi M, Del Guerra DA, Braga CP, Lucena FS, Silva JAM. Atividades educativas de trabalhadores da atenção primária: concepções de educação permanente e educação continuada em saúde presentes no cotidiano de Unidades Básicas de Saúde em São Paulo. Interface (Botucatu). 2009; 13(30):121-34

18. Demers AL, Mamary E, Ebin VJ. Creating opportunities for training California's public health workforce. J Contin Educ Health Prof. 2011; 31(1):64-9

19. Barbosa VBA, Ferreira MLSM, Barbosa PMK. Educação permanente em saúde: uma estratégia para a formação dos agentes comunitários de saúde. Rev Gaucha Enferm. 2012; 33(1):56-65.

20. Lemos SG, Morita MC, Carloto EMT. Recursos utilizados por graduandos em odontologia e cirurgiões dentistas do SUS para estudar e resolver dúvidas surgidas durante o trabalho. Revista ABENO. 2014; 14(1):3-16.

21. Ceccim RB, Feuerwerker LCM. O quadrilátero da formação para a área da saúde: ensino, gestão, atenção e controle social. Physis Rev. Saúde Coletiva. 2005; 14(1):41-65.

22. Ceccim RB, Bravin FP, Santos AA. Educação na saúde, saúde coletiva e ciências políticas: uma análise da formação e desenvolvimento para o Sistema Único de Saúde como política pública. Rev Lugar Comum. 2011; 28:159-80.

23. Barbosa GC, Meneguim S, Lima SAM, Moreno V. Política Nacional de Humanização e formação dos profissionais de saúde: revisão integrativa. Rev Brasil Enferm. 2013; 66(1):123-27.

24. Minas Gerais. Atenção em Saúde Bucal. Saúde em casa. Secretaria de Estado de Saúde de Minas Gerais. Belo Horizonte; 2006.

25. Paraná. Rede de Saúde Bucal. Secretaria da Saúde do Paraná. Curitiba; 2014.

26. Brasil. Decreto ${ }^{\circ} 6.286$ de 05 de dezembro de 2007 instituiu o Programa Saúde na Escola (PSE) e suas finalidades. Brasília, Ministério da Saúde; 2007.

27. Santiago LM, Rodrigues MTP, Júnior ADO, Moreira TMM. Implantação do programa saúde na escola em Fortaleza-CE: atuação de equipe da estratégia de saúde da família. Rev Brasil Enferm. 2012; 65(6):1026-29.

28. Linhares PHA, Lira GV, Albuquerque IMN. Avaliação do programa nacional de melhoria do acesso e da qualidade da atenção básica no estado do Ceará. Saúde Debate. 2014; 38(n. esp.):195-208.

29. Rodriguez LA, Vieira JDM, Leite ICG. Avaliação do fluxo de referência para um centro de especialidades odontológicas implantado em cidade de médio porte na região Sudeste. Cad Saude Colet. 2013; 21(1):40-5.

Correspondência para:

Maria Eneide Leitão de Almeida

e-mail: eneideufc@hotmail.com

Curso de Odontologia da UFC

Rua Alexandre Baraúna, 949, Rodolfo Teófilo

60430-160, Fortaleza/CE 